\title{
Low birth weight is associated with NIDDM in discordant monozygotic and dizygotic twin pairs
}

\author{
P. Poulsen ${ }^{1,2}$, A . A . Vaag ${ }^{1}$, K . O. Kyvik ${ }^{2}$, D. Møller Jensen ${ }^{1}$, H . B eck-N ielsen ${ }^{1}$ \\ ${ }^{1}$ Odense University Hospital, Department of Endocrinology and Internal Medicine M, Odense, Denmark \\ ${ }^{2}$ Genetic Epidemiology Research Unit, Odense University, Odense, Denmark
}

Summary Previous studies have demonstrated an association between low weight at birth and risk of later development of non-insulin-dependent diabetes mellitus (NIDDM). It is not known whether this association is due to an impact of intrauterine malnutrition per se, or whether it is due to a coincidence between the putative "NIDDM susceptibility genotype" and a genetically determined low weight at birth. It is also unclear whether differences in gestational age, maternal height, birth order and/or sex could explain the association. Twins are born of the same mother and have similar gestational ages. Furthermore, monozygotic (MZ) twins have identical genotypes. Original midwife birth weight record determinations were traced in $\mathrm{MZ}$ and dizygotic (DZ) twins discordant for NIDDM. Birth weights were lower in the NIDDM twins $(n=2 \times 14)$ compared with both their identical (MZ; $n=14)$ and non-identical (DZ; $n=$ 14) non-diabetic co-twins, respectively (MZ: mean \pm SEM $2634 \pm 135$ vs $2829 \pm 131$ g, p < 0.02; DZ: $2509 \pm$ 135 vs $2854 \pm 168 \mathrm{~g}, \mathrm{p}<0.02)$. Using a similar approach in $39 \mathrm{MZ}$ and DZ twin pairs discordant for impaired glucose tolerance (IGT), no significantly lower birth weights were detected in the IGT twins compared with their normal glucose tolerant cotwins. However, when a larger group of twins with different glucose tolerance were considered, birth weights were lower in the twins with abnormal glucose tolerance (NIDDM + IGT; $\mathrm{n}=106 ; 2622 \pm 45 \mathrm{~g}$ ) and IGT $(n=62: 2613 \pm 55 \mathrm{~g})$ compared with twins with normal glucose tolerance $(n=112: 2800 \pm 51 \mathrm{~g}$; $p=0.01$ and $p=0.03$, respectively). Furthermore, the twins with the lowest birth weights among the two co-twins had the highest plasma glucose concentrations $120 \mathrm{~min}$ after the 75 -g oral glucose load $(n=86$ pairs: $9.6 \pm 0.6$ vs $8.0 \pm 0.4 \mathrm{mmol} / \mathrm{l}, \mathrm{p}=0.03$ ). In conclusion, the association between low birth weight and NIDDM in twins is at least partly independent of genotype and may be due to intrauterine malnutrition. IGT was also associated with low birth weight in twins. However, the possibility cannot be excluded that the association between low birth weight and IGT could be due to a coincidence with a certain genotype causing both low birth weight and IGT in some subjects. [Diabetologia (1997) 40: 439-446]

Keywords Twins, non-insulin-dependent diabetes mellitus, birth weight, intrauterine malnutrition.
Received: 3 October 1996 and in revised form: 18 December 1996

Corresponding author: Dr. A. Vaag, Odense University Hospital, Department of Endocrinology and Internal Medicine M, Sdr. Boulevard, Odense, DK-5000 C, Denmark; E-mail: ALVA@post3.TELE.DK

A bbreviations: NIDDM, Non-insulin-dependent diabetes mellitus; MZ, monozygotic; DZ, dizygotic; IGT, impaired glucose tolerance; OGTT, oral glucose tolerance test; NGT, normal glucose tolerance. AUC, area under curve.
Studies from Hertfordshire and Preston in the United Kingdom consistently reported a higher prevalence of non-insulin-dependent diabetes mellitus (NIDDM) and/or impaired glucose tolerance (IGT) among subjects aged 50 to 70 years with either low birth weight and/or low weight at the age of 1 year $[1,2]$. The authors therefore suggested a major role of intrauterine malnutrition for the risk of developing NIDDM much later in life (i.e. after the age of 40 years). The association between low birth weight and risk of NIDDM has also been confirmed in 
Pima Indians [3] and recently in a Swedish population [4].

However, another study from Oxfordshire - also in the United Kingdom - did not demonstrate lower birth weights in either NIDDM patients or subjects with IGT; and concluded that genetic and "postnatal" environmental factors play quantitatively more important roles in the development of NIDDM compared with intrauterine malnutrition or low birth weight per se [5]. Accordingly, several epidemiological and metabolic studies of twins and first-degree relatives of NIDDM patients have indicated an important genetic component of NIDDM [6-10]. A postnatal environmental component is indicated by a strong association between the occurrence of NIDDM and the propagation of a modern western lifestyle [11]. Furthermore, the association between low birth weight and risk of NIDDM in some studies could theoretically be explained by a genetically determined reduced fetal growth rate. Thus, a reduced enhancement of growth in utero could - at least theoretically - be due to a putative genetically determined reduced insulin secretion or action in individuals predisposed to NIDDM $[4,5,10,12]$. In other words, the genotype responsible for NIDDM may itself cause retarded fetal growth in utero. The notion of the important influence of genetics on the weight at birth is supported by the recent demonstration of familial aggregation of low birth weight among both whites and blacks in the United States [13].

Other factors influencing birth weight include gestational age, maternal height, birth order and sex [13]; and correction for those factors in previous studies was either not performed or was associated with various degrees of uncertainty $[1,2,4,5,14]$.

Studying birth weights in twins discordant for NIDDM allows perfect matching for both gestational age, maternal height, birth order (in relation to other siblings) and sex. However, specifically studying identical (monozygotic or MZ) twins discordant for NIDDM allows elimination of a putative influence of the genotype per se on the fetal growth rate. Therefore, the aim of the present study was to trace birth weights in MZ and dizygotic (DZ) twins discordant for NIDDM and IGT respectively, and thereby to provide further insight into the possible role of low birth weight and of intrauterine malnutrition in the aetiology and pathophysiology of NIDDM and/ or IGT in twins.

\section{Subjects and methods}

Subjects. The twins were identified through the Danish Twin Register [15] and were residents of almost all areas of Denmark. Initially, questionnaires were sent out to a total of 3074 MZ and DZ twins aged 55 to 74 years during November 1994. Questionnaires were only sent out to twin pairs both of whom were of the same gender. The twins were asked whether they suffered from diabetes diagnosed after the age of 40 years and whether they would agree to participate in the present study involving a standard oral glucose tolerance test (OGTT) and anthropometric measurements (see below). A total of 975 twin pairs replied, and among those twins 303 twin pairs (= 606 twins) answered that they would both agree to participate in the study. Thus, twin pairs in whom only one of the two co-twins agreed to participate were not included in the study. Classification of twins (identical (MZ) vs non-identical (DZ)) was based on the similarity method [16, 17]. OGTTs were subsequently performed in the 303 twin pairs of whom 125 twin pairs were $\mathrm{MZ}$ and 178 twin pairs DZ. Based on the results from the OGTT and in accordance with the diagnostic criteria as outlined below, we identified a total of 45 twin pairs discordant for NIDDM (i.e. one twin with NIDDM and the other with non-diabetic glucose tolerance; $18 \mathrm{MZ}$ and $27 \mathrm{DZ}$ pairs), and a total of 17 pairs concordant for NIDDM (both twins with NIDDM; $9 \mathrm{MZ}$ and $8 \mathrm{DZ}$ pairs). Furthermore, 74 twin pairs were discordant for IGT (i.e. one twin with impaired and the other with normal glucose tolerance (NGT); $22 \mathrm{MZ}$ vs $52 \mathrm{DZ}$ pairs) and 19 pairs were concordant for IGT (both twins IGT; $9 \mathrm{MZ}$ vs $10 \mathrm{DZ}$ pairs).

Original midwife birth weight records were traced primarily in the twin pairs discordant for NIDDM according to predefined criteria for NIDDM as outlined below. Thus, as outlined in the Introduction, we considered this to be an optimal design to evaluate a possible influence of the intrauterine environment on the risk for later development of NIDDM. Using this approach, the group of "non-diabetic" twins included subjects with IGT. However, to gain further insight into a possible influence of the intrauterine environment on adult glucose tolerance, and especially on the state of "IGT" per se, we also traced available original midwife birth weight records for the additional twin pairs. Birth weight data originally recorded by the attending midwives were subsequently obtained from the mothers' delivery records, which in turn were traced through the various regional archives located in the individual areas throughout Denmark where birth was reported by the twins. Original midwives' records, including valid birth weight information, were available primarily for twins born after 1927. The records contained obstetric data on first- and secondborn twins, respectively. Classification of the twins as first- or second-born was based on information from the participating twins concerning the birth sequence. There was complete agreement concerning individual birth sequences within all twin pairs when asking each twin separately.

The protocol was approved by the regional ethical committee and the procedures were performed according to the principles of the Helsinki Declaration.

M ethods. All subjects participated in a standard 75-g OGTT after a 10-12-h overnight fast. Plasma glucose concentrations were analysed in the fasting state $(0 \mathrm{~min}), 30$ and $120 \mathrm{~min}$ after oral glucose ingestion by the glucose dehydrogenase oxidation method. Plasma insulin, proinsulin and C-peptide concentrations were measured using two-site, two-step, time resolved immunofluorometric assays as previously described (DELFIA) $[18,19]$. Plasma insulin concentrations were measured at the same time points as the plasma glucose concentrations, whereas plasma proinsulin and C-peptide concentrations were measured only in the fasting state. Incremental glucose and insulin areas under the curves (AUCs) were calculated using the trapezoidal method. Cross reactivities with proinsulin, C-peptide and Des(31,32)-split product in the insulin assay were all less than $0.4 \%$. Intra-assay coefficients of variation in the physiological ranges were $3.6-4.3 \%$ for plasma insulin; 
Table 1. Clinical characteristics of the twin pairs discordant for NIDDM

\begin{tabular}{|c|c|c|c|c|}
\hline & \multicolumn{2}{|c|}{ Monozygotic twins } & \multicolumn{2}{|l|}{ Dizygotic twins } \\
\hline & NIDDM & Non-diabetic & NIDDM & Non-diabetic \\
\hline $\mathrm{n}$ (females/males) & $14(7 / 7)$ & $14(7 / 7)$ & $14(7 / 7)$ & $14(7 / 7)$ \\
\hline Weight (kg) & $83 \pm 4^{a}$ & $72 \pm 5$ & $71 \pm 3$ & $74 \pm 4$ \\
\hline Height $(\mathrm{cm})$ & $165 \pm 3$ & $164 \pm 3$ & $165 \pm 3$ & $166 \pm 3$ \\
\hline $\mathrm{BMI}\left(\mathrm{kg} / \mathrm{m}^{2}\right)$ & $30.6 \pm 1.43^{\mathrm{a}}$ & $26.8 \pm 1.5$ & $26.2 \pm 1.2$ & $26.8 \pm 1.3$ \\
\hline Waist/hip ratio & $0.95 \pm 0.01^{\mathrm{a}}$ & $0.91 \pm 0.02$ & $0.90 \pm 0.02$ & $0.86 \pm 0.03$ \\
\hline 2-h OGTT glucose (mmol/l) & $18.4 \pm 1.2^{\mathrm{a}}$ & $7.9 \pm 0.4$ & $14.8 \pm 1.7^{\mathrm{a}}$ & $6.7 \pm 0.4$ \\
\hline Fasting insulin $(\mathrm{pmol} / \mathrm{l})$ & $79 \pm 9^{b}$ & $43 \pm 2$ & $71.4 \pm 13$ & $50.5 \pm 6.6$ \\
\hline 30 min OGTT insulin $(\mathrm{pmol} / \mathrm{l})$ & $172 \pm 29^{d}$ & $248 \pm 49$ & $210 \pm 41^{\mathrm{d}}$ & $356 \pm 79$ \\
\hline 120-min OGTT insulin (pmol/1) & $313 \pm 77$ & $298 \pm 34$ & $247 \pm 57$ & $388 \pm 56$ \\
\hline Glucose AUC $(\min \times \mathrm{mmol} / \mathrm{l})$ & $635 \pm 42^{\mathrm{a}}$ & $326 \pm 37$ & $633 \pm 88^{\mathrm{a}}$ & $253 \pm 33$ \\
\hline Insulin AUC $(\min \times \mathrm{pmol} / \mathrm{l})$ & $16124 \pm 4454$ & $23745 \pm 3780$ & $16216 \pm 4146^{\mathrm{c}}$ & $33519 \pm 5814$ \\
\hline Cholesterol (mmol/l) & $5.7 \pm 0.3$ & $5.8 \pm 0.3$ & $5.9 \pm 0.3$ & $6.4 \pm 0.4$ \\
\hline HDL cholesterol (mmol/l) & $1.11 \pm 0.09$ & $1.34 \pm 0.12$ & $1.43 \pm 0.11$ & $1.68 \pm 0.15$ \\
\hline LDL cholesterol (mmol/l) & $3.68 \pm 0.28$ & $3.87 \pm 0.24$ & $3.83 \pm 0.33$ & $4.14 \pm 0.38$ \\
\hline Birth weight $(\mathrm{g})$ & $2634 \pm 135^{\mathrm{c}}$ & $2829 \pm 131$ & $2509 \pm 135^{\mathrm{c}}$ & $2854 \pm 168$ \\
\hline
\end{tabular}

Mean \pm SEM; $n=14$ for all determinations except for the plasma lipid concentrations where $n=12$. All measurements were in plasma or serum samples. ${ }^{a} p<0.001,{ }^{b} p<0.01,{ }^{c} p<0.02$,
${ }^{\mathrm{d}} \mathrm{p}<0.05$; NIDDM vs non-diabetic co-twins. ${ }^{\mathrm{e}} \mathrm{p}<0.05$; non-diabetic MZ vs non-diabetic DZ twins
4.1-6.4\% for plasma proinsulin; and $1.7-2.3 \%$ for plasma Cpeptide concentrations. Inter-assay coefficients of variation were $1.7-3.4 \%$ for plasma insulin; $5.7-7.2 \%$ for plasma proinsulin; and $2.7-6.3 \%$ for plasma C-peptide concentrations. The fasting blood samples were also analysed for serum triglycerides, total cholesterol, HDL-cholesterol and LDL-cholesterol using commercial kits from Boerhinger Mannheim, Mannheim, Germany. Plasma lipid concentrations were only measured in the twins discordant for NIDDM. Waist circumference was measured using a soft tape on standing subjects midway between the lowest rib and the iliac crest. Hip circumference was measured over the widest part of the gluteal region, and the waist-to-hip ratio was calculated accordingly.

Criteria for the diagnosis of NIDDM included 1) diagnosis of diabetes after the age of 40 years and current use of antidiabetic medication or diet and/or 2) according to the World Health Organization (WHO) criteria (i.e. fasting venous plasma glucose concentration $\geq 7.8 \mathrm{mmol} / \mathrm{l}$ and/or $120 \mathrm{~min}$ venous plasma glucose concentration $\geq 11.1 \mathrm{mmol} / \mathrm{l}$ after a 75 -g oral glucose load. Subjects with fasting plasma glucose concentrations below $7.8 \mathrm{mmol} / \mathrm{l}$ and $120 \mathrm{~min}$ OGTT plasma glucose concentrations $\geq 7.8 \mathrm{mmol} / \mathrm{l}$ and $<11.1 \mathrm{mmol} / \mathrm{l}$ were classified as having IGT according to WHO criteria.

\section{Statistical analysis}

Non-parametric statistical methods (Wilcoxon's signed rank test for paired data (i.e. within twin pair differences) and Mann-Whitney test for unpaired data (i.e. between twin pair differences) were used for analysis of data. Correlation analyses were performed using the non-parametric Spearmans rho (R) analysis. All tests applied were two-tailed and $p=0.05$ or less was considered significant. Data are presented as mean \pm SEM.

\section{Results}

Complete birth weight records were identified for 28 pairs (i.e. 56 individual twins) out of the total number of 45 twin pairs who were discordant for NIDDM at the time of the present study. Fourteen of the 28 pairs were MZ (identical) and 14 were DZ (non-identical) twin pairs.

Clinical characteristics of the 28 twin pairs discordant for NIDDM are given in Table 1. Nine of the MZ and 8 of the DZ NIDDM twins were known NIDDM patients at the time when OGTTs were performed. Mean duration of diabetes in those twins was 9 (range 1-32) years for the $\mathrm{MZ}$ and 11 (range 2-20) years for the DZ twins. One of the MZ NIDDM twins was treated with insulin, 6 with oral hypoglycaemic agents and 2 with diet. Four of the DZ NIDDM twins were treated with insulin, 1 with an oral hypoglycaemic agent and 3 with diet. Treatment was withdrawn at least $24 \mathrm{~h}$ before the OGTT. The NIDDM diagnosis was confirmed in all of the previously diagnosed NIDDM twins according to the results of the OGTTs and WHO criteria. The rest of the NIDDM twins (i.e. 5 of the MZ and 6 of the DZ NIDDM twins) were newly diagnosed NIDDM patients based on the results of OGTTs in accordance with the WHO criteria as described, and were therefore not undergoing any treatment at the time of this study.

The mean age was similar in MZ and DZ twins (Table 1). Compared with their non-diabetic cotwins, the NIDDM MZ twins were significantly 
heavier, had higher body mass indices and showed more abdominal obesity (Table 1). The NIDDM and non-diabetic DZ twins did not differ significantly in weight, BMI or waist-to-hip ratios (Table 1). Both MZ and DZ diabetic twins had significantly higher fasting and 2-h plasma glucose compared with their non-diabetic co-twins (Table 1). Furthermore, the non-diabetic MZ twins had significantly higher mean fasting plasma glucose concentrations compared with the non-diabetic DZ twins (Table 1). Seven of the 14 non-diabetic MZ twins and 4 of the 14 non-diabetic DZ twins had IGT according to WHO criteria. Incremental glucose AUCs during the OGTT mainly reflected the 120-min plasma OGTT glucose concentrations, and were higher in diabetic compared with non-diabetic twins (Table 1). Incremental insulin AUCs were lower in diabetic twins compared with their non-diabetic co-twins, although this difference only reached statistical significance in the DZ twins (Table 1). The ratio between incremental insulin and glucose AUCs were lower in diabetic compared with their non-diabetic co-twins. In addition, the ratio between incremental insulin and glucose AUCs were lower in MZ non-diabetic compared with the DZ non-diabetic twins (Table 1). Serum triglycerides were higher in the MZ NIDDM twins compared with the MZ non-diabetic twins (Table 1). Serum total cholesterol concentrations were similar in all groups, and serum HDL - and LDL cholesterol concentrations did not differ significantly between the study groups (Table 1). Finally, blood pressure measurements were not significantly different between any of the study groups (data not shown).

Individual intra-pair birth weight differences and mean birth weights in the twins discordant for NIDDM are presented in Figure 1 and in Table 1. The range of individual birth weights were for $\mathrm{MZ}$ NIDDM twins: $1550-3500 \mathrm{~g}$; MZ non-diabetic twins: 1850-3500 g; DZ NIDDM twins: 1500-3250 g; DZ non-diabetic twins: $2000-4600 \mathrm{~g}$. The twins with documented NIDDM had significantly lower birth weights compared with their non-diabetic co-twins irrespective of zygosity classification $(2571 \pm 95$ vs $2841 \pm$ $105 \mathrm{~g}, \mathrm{p}<0.0001)$. Furthermore, when the two twin groups were regarded separately, the lower birth weights in the NIDDM twins compared with their non-diabetic co-twins remained statistically significant for both MZ $(2634 \pm 135$ vs $2829 \pm 131 \mathrm{~g}, \mathrm{p}<$ $0.02)$ and DZ $(2509 \pm 135$ vs $2854 \pm 168 \mathrm{~g}, \mathrm{p}<0.02)$ twin pairs (Table 1, Figure 1). Finally, when the twins $(\mathrm{n}=28$ pairs) were divided into pairs with NGT according to WHO criteria in the non-diabetic twins ( $n=17$ pairs), and pairs with IGT in the non-diabetic twins ( $\mathrm{n}=11$ pairs), the NIDDM twins had statistically significant lower birth weights compared with both their NGT and IGT co-twins, respectively (NIDDM vs NGT co-twins: $2587 \pm 124$ vs $2827 \pm$ $162 \mathrm{~g}, \mathrm{p}<0.05$; and NIDDM vs IGT-co-twins: $2548 \pm$
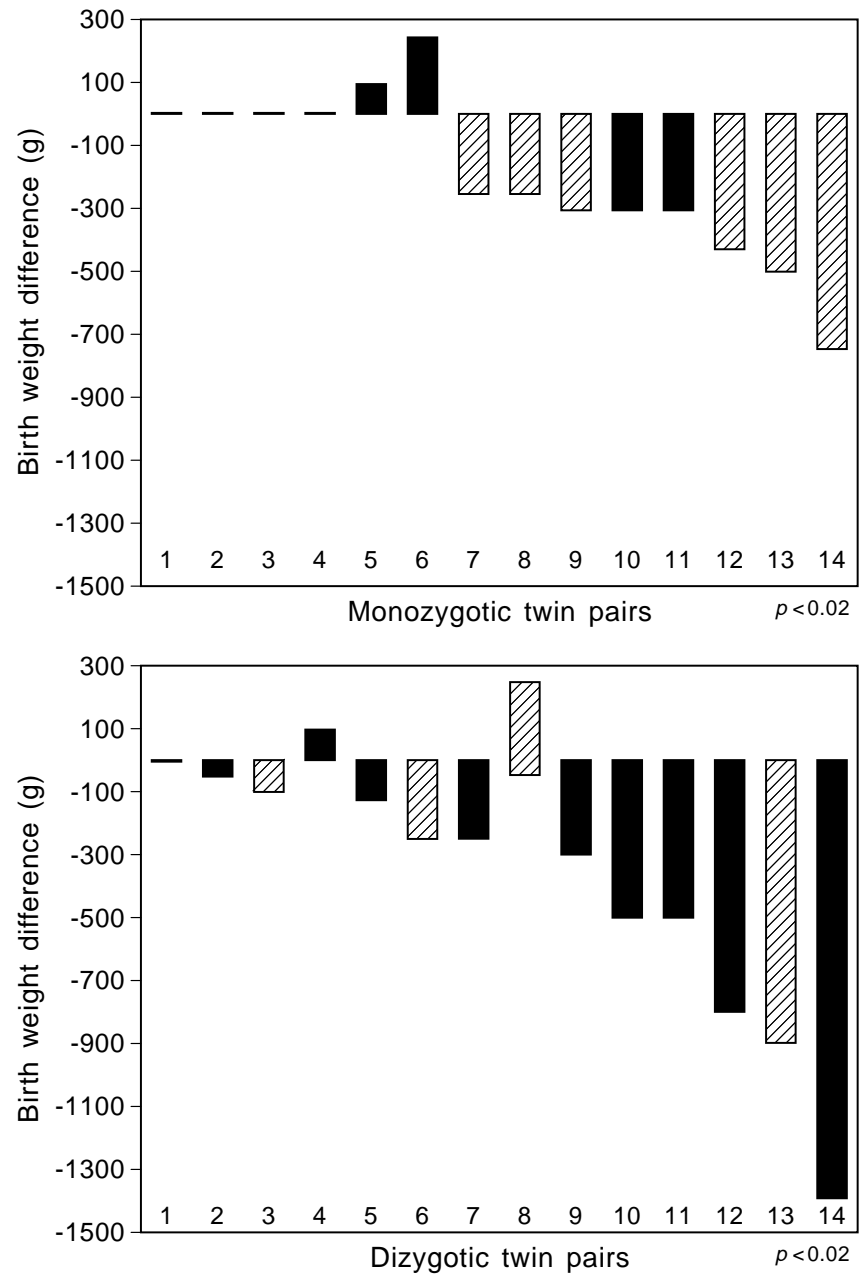

Fig. 1. Individual intra-pair birth weight differences in the dizygotic (DZ; non-identical) and monozygotic (MZ; identical) twin pairs discordant for NIDDM (i.e. birth weight of the NIDDM twin minus birth weight of the corresponding non-diabetic co-twin). Note that a negative value is obtained when the birth weight of the NIDDM twin is lower than the birth weight of the corresponding non-diabetic co-twin. The intrapair birth weight differences are presented in a "ranked" order according to the absolute magnitude of the differences. Hatched bars indicate IGT in the "non-diabetic twin" $(n=11$ twin pairs including one pair with similar birth weights among the two co-twins), whereas closed bars indicate normal glucose tolerance in the non-diabetic twin according to the WHO criteria $(n=17$ twin pairs including 4 pairs with similar birth weights among the two co-twins). MZ and DZ NIDDM vs non-diabetic co-twins: $p<0.02$ for both comparisons

153 vs $2864 \pm 102 \mathrm{~g}, \mathrm{p}<0.01)$. No significant correlation was found between the duration of diabetes and intra-pair differences in birth weights in the discordant pairs.

Additional original birth weight records were traced in 81 twin pairs out of the 303 pairs who participated in the OGTT. Of those, 8 pairs (4 MZ and 4 DZ pairs) were concordant for NIDDM, 6 pairs (3 $\mathrm{MZ}$ and $3 \mathrm{DZ}$ pairs) were concordant for IGT, 39 pairs (13 MZ and $26 \mathrm{DZ}$ pairs) were discordant for IGT, and in 28 pairs both twins were non-diabetic. 
Table 2. Clinical characeristics of the twin pairs $(M Z+D Z)$ differing in birth weight

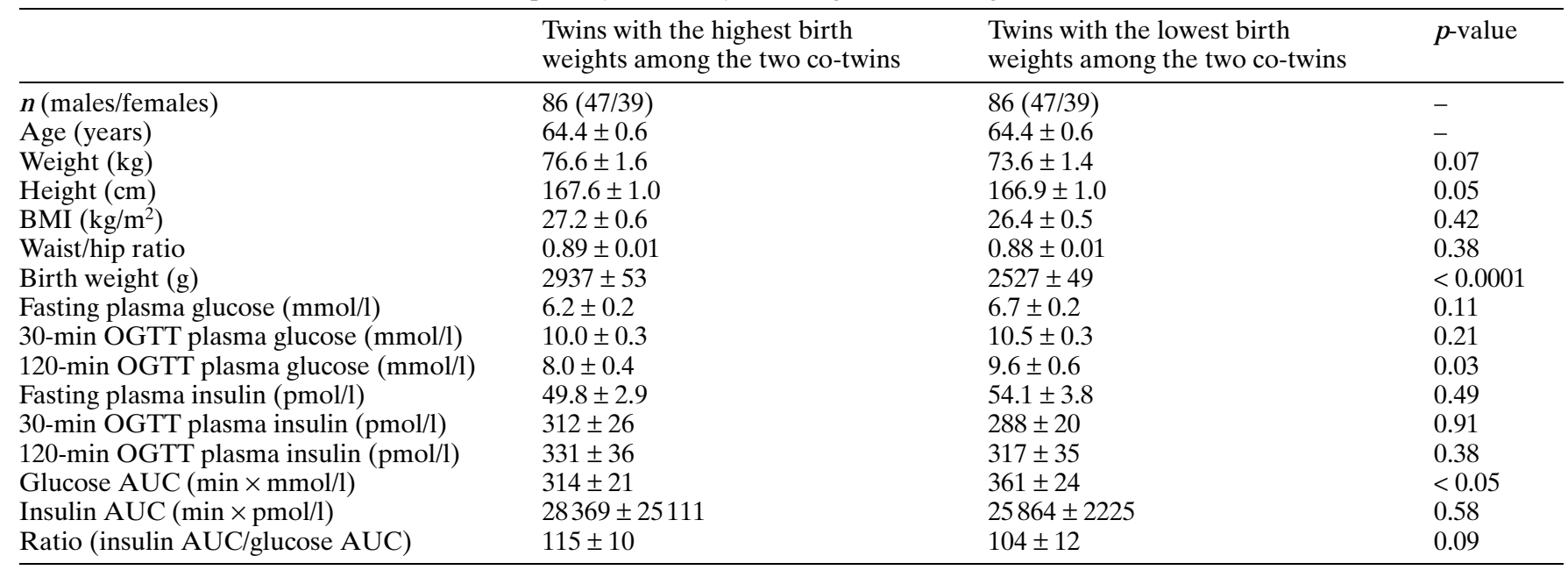

No significant difference in birth weights was detected within the concordant twin pairs among the two co-twins who reported to have diabetes diagnosed first compared with second or last (ie first vs second diagnosed diabetes; mean birth weights: $2800 \pm 240$ vs $2750 \pm 185 \mathrm{~g}$, NS). The difference in duration of "known" diabetes within the concordant twin pairs was $5 \pm 1$ years.

No significant differences in birth weights were detected within twin pairs discordant for IGT when the twin pairs were considered as one group $(n=39 \mathrm{MZ}$ and DZ twin pairs; IGT twins vs NGT co-twins: $2604 \pm 70$ vs $2623 \pm 65 \mathrm{~g}$, NS) or separately as MZ $(n=13)$ or DZ $(n=26)$ twins (data not shown). However, in this larger group of both MZ and DZ twins $(\mathrm{n}=218$ twins $(=109$ twin pairs $)$, birth weights were significantly lower in the combined group of twins with either NIDDM or IGT compared with twins with NGT $(n=106$ vs 112 ; mean \pm SEM: $2622 \pm 45$ vs $2800 \pm 51 \mathrm{~g}, \mathrm{p}=0.01$ ). In addition, mean birth weights were significantly lower in the total group of $\mathrm{MZ}$ and $\mathrm{DZ}$ twins with IGT compared with the twins with NGT $(n=62$ vs 112 ; mean \pm SEM: $2613 \pm 55$ vs $2800 \pm 51 \mathrm{~g}, \mathrm{p}=0.03$ ).

Individual birth weights did not correlate significantly with fasting, 30-min or 120-min OGTT plasma glucose concentrations in the twins discordant for NIDDM. However, considering all 218 individual twins with known birth weights, individual twin birth weights correlated inversely with the 120-min OGTT plasma glucose concentration $(n=218, R=-0.15$, $p<0.03$ ). Furthermore, a somewhat stronger correlation was found between birth weight and 120-min OGTT plasma glucose concentration, when individual intra-pair difference correlations were determined $(n=109$ pairs, $R=-0.26, p<0.01)$.

A statistically significant positive correlation was found between birth weight and adult body weight $(n=218, R=0.14, p=0.03)$, and a negative correlation was found between birth weight and 120-min OGTT plasma insulin concentration $(\mathrm{n}=$ $218, R=-0.13, p<0.05$ ). No statistically significant correlations were determined between individual birth weights and fasting plasma glucose concentration, 30-min OGTT plasma glucose concentrations, BMIs or waist-to-hip ratios. Furthermore, no statistically significant correlations were found between individual birth weights and fasting or 30-min OGTT plasma insulin, or between birth weights and fasting proinsulin or C-peptide concentrations in the total group of 109 twin pairs ( = 218 individual twins), or in any study group sub-populations. Finally, birth weights did not correlate significantly with incremental insulin AUCs, glucose AUCs or with the ratio between insulin AUCs and glucose AUCs.

In 23 twin pairs out of the total of $109 \mathrm{MZ}$ and DZ twin pairs, the two co-twins had similar birth weights. In the remaining 86 twin pairs - including both normal, IGT and NIDDM twins - the twins with the lowest birth weights among the two co-twins had significantly higher 120-min OGTT plasma glucose concentrations and incremental glucose AUC during OGTT compared with their co-twins with the highest birth weights (Table 2$)$. When MZ ( $n=36$ pairs) and DZ $(n=50$ pairs $)$ were considered separately, the difference in 120-min OGTT plasma glucose concentrations reached statistical significance in the $\mathrm{MZ}$ twins $(p<0.05)$, but not in the DZ twins $(p=0.12)$. Incremental glucose AUCs were not significantly different between twins with the lowest and highest birth weights when $\mathrm{MZ}$ and $\mathrm{DZ}$ twins were considered separately $(p=0.25$ and 0.19 , respectively). Fasting or 30-min OGTT plasma glucose concentrations were not significantly higher in the twins with the lowest compared with the highest birth weights (Table 2). However, adult height was slightly but significantly lower in the twins with the lowest compared with highest birth weights (Table 2). 
Furthermore, the absolute adult weight was lower in twins with the lowest birth weights, although this difference did not reach statistical significance (Table 2). Finally, when comparing twins with the highest and lowest birth weights among the two co-twins with different birth weights, we were unable to detect any significant effect of birth weight on BMI, waistto-hip ratio, fasting 30-min or 120-min plasma insulin concentrations, incremental insulin AUC, or on the incremental (insulin AUC/glucose AUC) ratio (Table 2).

\section{Discussion}

The finding of significantly lower birth weights in both MZ and DZ NIDDM twins compared with their non-diabetic co-twins excludes the possibility that other factors such as gestational age, maternal height, birth order and/or sex [20] could explain the lower birth weights in the NIDDM patients/twins in this and perhaps in previous studies. Most importantly, however, the finding of lower birth weights in the MZ NIDDM twins compared with their genetically identical non-diabetic co-twins eliminates the theoretical possibility that the association between low birth weight and risk of NIDDM could be explained solely by a coincidence between the "NIDDM susceptibility genotype" and impaired intrauterine growth. Therefore, the lower birth weights in the twins who later developed NIDDM were probably due to some degree of intrauterine malnutrition, at least compared with their co-twins who did not develop diabetes. Finally, the impact of low birth weight on diabetic status is supported by our finding in the larger group of unselected twin pairs $(n=86$ twin pairs), that twins with the lowest birth weight among the two co-twins had a significantly higher 120-min OGTT plasma glucose concentration, and a significantly higher incremental glucose AUC during the OGTTs (Table 2).

It is noteworthy that we were able to detect a statistically significant mean difference of only $195 \mathrm{~g}$ in birth weights within the relatively small group of 14 MZ twin pairs discordant for NIDDM (Table 1, Figure 1). Specifically, this small but significant difference is thought-provoking when seen in the light of the large range of birth weights from 1500 to $3500 \mathrm{~g}$ in the group of NIDDM twins. This may suggest that it is not so much an absolute low birth weight per se, but rather a disproportionately low birth weight in relation to an individual and possibly to a large extent genetically determined birth weight, which predispose to NIDDM much later in life. Moreover, it may also illustrate that low birth weight is possibly only a marker of an unfavourable intrauterine environment or event predisposing to NIDDM, rather than a predisposing factor to NIDDM itself.
The notion that birth weight may at least be partly genetically determined is in accordance with the recent study by Wang et al. [13]. It may also explain why large study materials were required to demonstrate an association between low birth weight and NIDDM in general population studies [1-3], and why at least one study did not demonstrate lower absolute birth weights in NIDDM patients [5]. However, when eliminating the genetic influence on birth weight using identical twins, which in addition eliminate the difficulties assessing and correcting for gestational age, maternal height, etc, small differences in a small study population can be detected.

The impact of a non-genetic intrauterine component for the risk of NIDDM such as malnutrition demonstrated in this and other studies [1-3], does not of course exclude that other factors including both "postnatal" environmental and genetic components may play important roles in the aetiology and pathophysiology of NIDDM. However, it does expand our conception of NIDDM as a multifactorial disease, and may also to some extent bring into question some conclusions concerning the aetiology of NIDDM made from previous studies. For one thing, the notion of an important genetic component in the aetiology of NIDDM is partly based on the finding of higher concordance rates of NIDDM in MZ compared with DZ twins [6, 7]. However, MZ twins are more likely to suffer from intrauterine malnutrition compared with DZ twins [21], and the validity of twin studies for aetiological conclusions concerning genes vs environment in diseases where intrauterine malnutrition may play a role (as in NIDDM) has therefore also been questioned [22]. On the other hand, the evidence for a genetic component in NIDDM also comes from several studies demonstrating metabolic abnormalities and a higher risk of NIDDM in offspring of NIDDM patients [9]. In addition, the risk of NIDDM differs significantly between different ethnic populations [23, 24] which further supports the existence of a "NIDDM susceptibility genotype" in the aetiology of NIDDM. In this study we found a higher fasting plasma glucose concentration, and a significantly lower ratio of incremental insulin-glucose AUC ratio during OGTTs in the group of non-diabetic MZ twins compared with the non-diabetic DZ twins in the face of virtually identical birth weights (Table 1). This, by itself, could be taken to support the existence of and a role for a NIDDM susceptibility genotype in the aetiology of NIDDM independent of birth weight and intrauterine malnutrition.

It should also be mentioned that the present study demonstrated the impact of a non-genetic intrauterine component in the twin in a given twin pair who developed diabetes first. Thus, the non-diabetic cotwins in (at least MZ twin pairs) discordant for NIDDM may themselves exhibit various degrees of 
prediabetic abnormalities of glucose metabolism [10, 25], and are therefore highly likely to develop NIDDM later in life [7].

Hales et al. [1] reported an association between low birth weight and abnormal glucose tolerance at age 64 years, including subjects with overt NIDDM and IGT in one group. In the present study, we did not find significantly lower birth weights in the 39 twins with IGT compared with their normal glucose tolerant MZ and DZ co-twins. Therefore, using a similar approach and study design as in the twin pairs discordant for NIDDM per se, we were unable to demonstrate any influence of a non-genetic intrauterine component - or even of low birth weight - in the aetiology and/or pathophysiology of IGT per se. On the other hand, after the inclusion of a larger number of twins concordant for either NIDDM, IGT or NGT, we did find statistically significant lower birth weights in this larger population of IGT twins compared with the total group of NGT twins. Therefore, our data does support the previous finding of an association between low birth weight and both IGT and NIDDM as originally reported by Hales et al. [1]. However, because we did not find any difference in birth weights within the MZ twin pairs discordant for IGT per se, we cannot exclude the theoretical possibility that the association between low birth weight and IGT may at least partly be due to a coincidence between a specific genotype responsible for both a genetically determined low birth weight and IGT in some subjects. For example, one such coincidence could be due to a mutation or polymorphism at the coding region for insulin receptor substrate-1 (IRS1 ), which in its presence may both cause low birth weight [26], insulin resistance [27] and an increased risk of developing NIDDM in adult age [28]. Finally, we cannot exclude that other factors such as gestational age, maternal height and/or birth order could have explained the association between low birth weight and IGT in twins using the unpaired study design.

The correlations between birth weight, plasma glucose and plasma insulin concentrations found in this study were relatively weak. In the case of 120 -min OGTT plasma glucose concentrations, the correlation coefficient of -0.26 indicates that birth weight per se may only explain $5 \%$ of the variance. Furthermore, various factors associated with the insulin resistance syndrome including BMI and waist-to-hip ratio did not correlate significantly with birth weight. This, by itself, supports the view that other factors including both genetics and postnatal environmental factors play important roles in the aetiology and pathophysiology of both abnormal glucose tolerance and insulin resistance in man.

The mechanism through which intrauterine malnutrition increases the risk of NIDDM may include both impaired insulin secretion [5] and peripheral insulin resistance $[4,29]$. The weak but significant inverse correlation between birth weight and 120-min OGTT plasma insulin concentrations found in this study may support a role of insulin resistance. However, studies using more sophisticated methods such as the hyperinsulinaemic euglycaemic clamp technique are required to provide more insight into this issue. Furthermore, the possibility that intrauterine malnutrition increases the risk of obesity in adult life, a known risk factor of NIDDM, cannot be totally excluded [30]. The latter possibility may to some extent be supported by the finding of a significantly higher adult weight and BMI in the MZ NIDDM twins compared with the MZ non-diabetic twins in this (Table 1) and in our previous study [10]. However, this is not likely to be the sole explanation as the DZ NIDDM and the DZ non-diabetic twins had similar adult body weights in the face of significantly lower birth weights in the DZ NIDDM twins (Table 1). Furthermore, we found a positive rather than a negative correlation between birth weight and adult weight in the total twin study population. Therefore, our results are in keeping with the previous conclusion that the association between low birth weight and NIDDM goes beyond a putative influence of low birth weight on adult body weight $[1,4]$.

In conclusion, the study supports the role of a nongenetic (environmental) intrauterine component, such as intrauterine malnutrition, for the development of NIDDM much later in life. IGT per se was also associated with low birth weight in twins. However, we cannot exclude that the latter association could at least to some extent be due to a coincidence with a certain genotype causing both a low birth weight and IGT in some subjects. An important challenge in future studies will be to address the exact nature of this non-genetic intrauterine factor in the aetiology of NIDDM, and to determine the extent to which this plays a role in the aetiology and pathophysiology of NIDDM as compared with genetics and postnatal environmental factors.

A cknowledgements. We are grateful to Ms. E. Beck-Nielsen and Ms. G. Hauge for their help during the process of tracing the twin pairs. Dr. J.Vinten is acknowledged for commenting the manuscript. The study was supported by grants from the Danish Medical Research Council, the Danish Diabetes Association, the NOVO Foundation, the Velux Foundation and from the Clinical Research Institute, Odense University Hospital.

\section{References}

1. Hales CN, Barker DJ, Clark PM et al. (1991) Fetal and infant growth and impaired glucose tolerance at age 64 . BMJ 303: 1019-1022

2. Phipps K, Barker DJ, Hales CN, Fall CH, Osmond C, Clark PM (1993) Fetal growth and impaired glucose tolerance in men and women. Diabetologia 36: 225-228 
3. McCance DR, Pettitt DJ, Hanson RL, Jacobsson LTH, Knowler WC, Bennett PH (1994) Birth weight and non-insulin dependent diabetes: thrifty genotype, thrifty phenotype, or surviving small baby genotype? BMJ 308: 942-945

4. Lithell HO, McKeigue PM, Berglund L, Mohsen R, Lithell U-B, Leon DA (1996) Relation of size at birth to non-insulin dependent diabetes and insulin concentrations in men aged 50-60 years. BMJ 312: 406-410

5. Cook JTE, Levy JC, Page RCL, Shaw JAG, Hattersley AT, Turner RC (1993) Association of low birth weight with beta-cell function in the adult first degree relatives of noninsulin-dependent diabetes subjects. BMJ 306: 302-306

6. Barnett AH, Eff C, Leslie RDG, Pyke DA (1981) Diabetes in identical twins. Diabetologia 20: 87-93

7. Newman B, Selby JV, King MC, Slemenda C, Fabsitz R, Friedman GD (1987) Concordance for type 2 (non-insulin-dependent) diabetes mellitus in male twins. Diabetologia 30: 763-768

8. Warram JH, Martin BC, Krolewski AS, Soeldner JS, Kahn CR (1990) Slow glucose removal rate and hyperinsulinemia precede the development of type II diabetes in the offspring of diabetic parents. Ann Intern Med 113: 909-915

9. Beck-Nielsen H, Groop L (1994) Metabolic and genetic characterisation of prediabetic states. Sequence of events leading to non-insulin-dependent diabetes mellitus. J Clin Invest 94: 1714-1721

10. Vaag A, Henriksen JE, Madsbad S, Holm N, Beck-Nielsen $H$ (1995) Insulin secretion, insulin action, and hepatic glucose production in identical twins discordant for NIDDM. J Clin Invest 95: 690-698

11. Zimmet P (1982) Type 2 (non-insulin-dependent) diabetes - an epidemiological overview. Diabetologia 22: 399-411

12. Gluckman PD (1986) The role of pituitary hormones, growth factors and insulin in the regulation of fetal growth. Oxf Rev Reprod Biol 8: 1-60

13. Wang X, Zuckerman B, Coffman GA, Corwin MJ (1995) Familial aggregation of low birth weight among whites and blacks in the United States. N Engl J Med 333: 1744-1749

14. Robinson S, Walton RJ, Clark PM, Barker DJ, Hales CN, Osmond C (1992) The relation of fetal growth to plasma glucose in young men. Diabetologia 35: 444-446

15. Hauge M, Harvald B, Fischer M et al. (1968) The Danish twin Register. Acta Genet Med Gemellol 17: 315-331

16. Gedda L (1966) The use of twins in epidemiological studies. Report of the WHO meeting of investigators on methodology of twin studies. Acta Genet Med Gemellol 15: 109-128
17. Magnus P, Berg K, Nance WE (1983) Predicting zygosity in Norwegian twin pairs born 1915-1960. Clin Genet 24: 103 112

18. Hemmilä I, Dakubu S, Mukkala V-M, Siitari H, Lövgren T (1984) Europium as a label in time-resolved immunofluorometric assays. Analytical Biochemestry 137: 335-343

19. Gjessing HJ, Reinholdt B, Pedersen O (1989) The plasma $\mathrm{C}$-peptide and insulin responses to stimulation with intravenous glucagon and a mixed meal in well-controlled type 2 (non-insulin-dependent) diabetes mellitus: dependency on acutely established hyperglycaemia. Diabetologia 32: 858-863

20. Tanner JM, Thomson AM (1970) Standards for birth weight at gestation periods from 32 to 42 weeks, allowing for maternal height and weight. Arch Dis Child 45: 566-569

21. Ramos-Arroyo MA, Ulbright TM, Yu TL, Christian JC (1988) Twin study: relationship between birth weight, zygosity, placentation, and pathologic placental changes. Acta Genet Med Gemellol 37: 229-238

22. Phillips DIW (1993) Twin studies in medical research: can they tell us whether diseases are genetically determined? Lancet 341: 1008-1009

23. West KM (1974) Diabetes in American Indians and other native populations of the new world. Diabetes 23: 841-855

24. Zimmet P (1979) Epidemiology of diabetes and its macrovascular manifestations in Pacific populations: the medical effects of social progress. Diabetes Care 2: 144-153

25. Barnett AH, Spiliopoulos AJ, Pyke DA, Stubbs WA, Burrin J, Alberti KGMM (1981) Metabolic studies in unaffected co-twins of non-insulin dependent diabetics. BMJ 282: $1656-1658$

26. Tamemoto H, Kadowaki T, Tobe K et al. (1994) Insulin resistance and growth retardation in mice lacking insulin receptor substrate-1. Nature 372: 182-186

27. Clausen JO, Hansen T, Bjorbaek C et al. (1995) Insulin resistance: interactions between obesity and a common variant of insulin receptor substrate-1. Lancet 346: 397-402

28. Almind K, Bjorbaek C, Vestergaard H, Hansen T, Echwald S, Pedersen O (1993) Aminoacid polymorphisms of insulin receptor substrate-1 in non-insulin-dependent diabetes mellitus. Lancet 342: 828-832

29. Phillips DIW, Barker DJP, Hales CN, Hirst S, Osmond C (1994) Thinness at birth and insulin resistance in adult life. Diabetologia 37: 150-154

30. Ravelli GP, Stein ZA, Susser MW (1976) Obesity in young men after famine exposure in utero and early infancy. $\mathrm{N}$ Engl J Med 295: 349-353 